Ergod. Th. \& Dynam. Sys. (1983), 3, 1-12

Printed in Great Britain

\title{
Hyperbolic behaviour of geodesic flows on manifolds with no focal points
}

\author{
KEITH BURNS \\ Department of Mathematics, Southern Illinois University, Carbondale, Illinois 62901
}

(Received 20 July 1982 and revised 4 January 1983)

Abstract. It is shown that the unit tangent bundle of a compact uniform visibility manifold with no focal points contains a subset of positive Liouville measure on which all the characteristic exponents of the geodesic flow (except in the flow direction) are non-zero. This completes Pesin's proof that the geodesic flow of such a manifold is Bernoulli.

\section{Preliminary remarks}

Anosov showed in the early 1960's that the geodesic flow on the unit tangent bundle of a compact Riemannian manifold with negative curvature has a dense orbit; that the closed orbits of the geodesic flow are dense in the unit tangent bundle; and that the geodesic flow is ergodic. Since then efforts have been made to extend these results to a wider class of manifolds. Eberlein and O'Neill [10] introduced the axiom of uniform visibility (defined in $\S 1$ ) as a suitable generalization of negative curvature. Eberlein [5], [6], [7] showed that the geodesic flow on a compact uniform visibility manifold has a dense orbit, and that the geodesic flow is topologically mixing if the manifold also has non-positive curvature. Later Pesin $[17],[18],[19]$ investigated the ergodic aspects of the problem using his extensive theory of non-uniform hyperbolicity. Pesin considered manifolds with no focal points (a generalization of non-positive curvature) satisfying uniform visibility. $\mathrm{He}$ almost proved that the geodesic flow of such a manifold is ergodic with respect to the smooth measure on the unit tangent bundle. He showed that if the set $\Lambda$, where the characteristic exponents of the geodesic flow (other than in the flow direction) are non-zero, has positive measure, then it has full measure and the geodesic flow is ergodic and even isomorphic to a Bernoulli flow. We prove that $\Lambda$ has positive measure. One of the main steps in the argument will be to show that the closed orbits of the geodesic flow are dense. Precise statements of our result and Pesin's theorem will be found at the end of $§ 1$.

Ballmann [1], [2] has studied manifolds with non-positive curvature satisfying a condition weaker than uniform visibility. $\mathrm{He}$ and Brin have shown that these conditions imply that the geodesic flow of a compact manifold is ergodic and Bernoulli. An earlier version of their result is contained in [3]. In $\S 4$ we give a proof of Ballmann \& Brin's theorem. 


\section{Introduction and statement of results}

We begin by summarizing the properties of manifolds with no focal points which will be needed later. Most of this theory can be found in [18]; [5], [8], [11] and [12] are also useful references.

Throughout $M$ will be a compact, connected, smooth $\left(C^{\infty}\right)$, $n$-dimensional Riemannian manifold without boundary. We shall write $H$ for the Riemannian universal cover of $M$ and identify $\pi_{1}(M)$ with the group of covering transformations, which are isometries of $H$. Geodesics wili always have unit speed. If $v$ is a unit vector in $M$ or $H$, let $\gamma_{v}$ be the unique geodesic with $\gamma_{v}^{\prime}(0)=v$. The geodesic flow $\Phi_{t}$ on the unit tangent bundle $S M$ is defined by

$$
\Phi_{t}(v)=\gamma_{v}^{\prime}(t) .
$$

Let $\mu$ be the Liouville measure on $S M$. The measure $\mu$ is $\Phi_{t}$-invariant.

We shall always assume that $M$ has no focal points. This means that if $Y(t)$ is a Jacobi field along a geodesic in $M$ with $Y(0)=0$, then $\|Y(t)\|$ is strictly increasing as $t \rightarrow \infty$. Geometrically this means that the universal cover $H$ has two properties. Firstly

$$
\exp _{p}: T_{p} H \rightarrow H
$$

is a diffeomorphism for every $p \in H$ (i.e. $M$ has no conjugate points $[14, \mathrm{pp} 133$, 201]). So there is a unique geodesic passing through any two distinct points in $H$. Secondly every geodesic ball in $H$ is strictly convex in the following sense [9, lemma 1]. We shall say that a set $C \subseteq H$ is convex if the geodesic segment joining any two points of $C$ lies inside $C$. We shall say that $C$ is strictly convex if in addition $C$ is the closure of an open set in $H$ with $\partial C$ a $C^{2}$-embedded hypersurface, and for any $p \in \partial C$ we have

$$
C \cap \exp _{p}\left(T_{p} \partial C\right)=\{p\} .
$$

Any manifold with non-positive curvature has no focal points.

Geodesics $\gamma$ and $\delta$ in $H$ are asymptotic (as $t \rightarrow \infty$ ) if $d(\gamma(t), \delta(t)$ ) is bounded as $t \rightarrow \infty$, and biasymptotic if $d(\gamma(t), \delta(t))$ is bounded as $t \rightarrow \pm \infty$. Geodesics in $M$ are asymptotic if they have lifts to $H$ that are asymptotic. An equivalence class of asymptotic geodesics in $H$ is called a point at infinity. One can show that if $p \in H$ and $v \in S H$ there is a unique $w \in S_{p} H$ with $\gamma_{w}$ asymptotic to $\gamma_{v}$, and $w$ depends continuously on $p$ and $v$. Using this one can identify the set $H(\infty)$ of all points at infinity with the sphere $S^{n-1}$ and construct a natural topology (the cone topology) on

$$
\bar{H}=H \cup H(\infty)
$$

so that it is a closed disc with $H(\infty)$ as boundary. Each geodesic $\gamma$ in $H$ has uniquely defined endpoints $\gamma(-\infty), \gamma(\infty) \in H(\infty)$ so that

$$
\gamma:[-\infty, \infty] \rightarrow \bar{H}
$$

is continuous. Covering transformations of $M$ extend to homeomorphisms of $\bar{H}$. The angle function $\Varangle_{p}(x, y)$ is continuous on

$$
\{(p, x, y) \in H \times \bar{H} \times \bar{H}: p \neq x, p \neq y\} .
$$


These results were proved when $M$ has non-positive curvature by Eberlein and O'Neill [10]. It is clear from [11], [12] and [18] that they still hold when $M$ has no focal points.

For $v \in S H$ define $L(v)$ to be the boundary of

where

$$
\bigcup_{t>0} D\left(\gamma_{v}(t), t\right)
$$

$$
D(p, r)=\{q \in H: d(p, q) \leq r\}
$$

then $L(v)$ is orthogonal to the geodesics asymptotic to $\gamma_{v}$ and intersects each of them exactly once, cf. $[12, \S 2]$. Let

$$
\vec{L}(v)=L(-v)
$$

so $\bar{L}(v)$ is orthogonal to the geodesics asymptotic to $\gamma_{v}$ as $t \rightarrow-\infty$. The hypersurfaces $L(v)$ and $\bar{L}(v)$ are called horospheres. Let

$$
B(v)=L(v) \cap \bar{L}(v) .
$$

Proposition 1.1. (i) $L(v), \bar{L}(v)$ are hypersurfaces $C^{2}$-embedded in $H$, $[12$, theorem 2(i)].

(ii) $L(v)$ and $\bar{L}(v)$ are tangent at points of $B(v)$ and their common normals are biasymptotic to $\gamma_{v}$. These are the only geodesics biasymptotic to $\gamma_{v},[\mathbf{1 2}$, proposition 4].

(iii) If $\gamma$ and $\delta$ are biasymptotic geodesics in $H$ and

$$
\delta(0) \in \boldsymbol{B}\left(\gamma^{\prime}(0)\right) \text { then } \boldsymbol{B}\left(\delta^{\prime}(0)\right)=\boldsymbol{B}\left(\gamma^{\prime}(0)\right) \text {. }
$$

(iv) If $v_{n} \rightarrow v$ in $S H, p_{n} \rightarrow p$ in $H$ and $p_{n} \in B\left(v_{n}\right)$ for each $n$, then $p \in B(v)$.

(v) $B(v)$ is convex, [12, theorem 2(ii)].

(vi) If $\gamma$ and $\delta$ are biasymptotic geodesics in $H$, then $d(\gamma(t), \delta(t))$ is constant, [16, proposition 4].

(vii) The Flat Strip Theorem: the geodesics $\gamma$ and $\delta$ in (vi) bound a strip of flat totally geodesically immersed surface, [12], [16].

Along each geodesic we have the families of stable and unstable Jacobi fields. They are constructed in [8], [12], [18] as certain limiting solutions of Jacobi's equation. We give an equivalent description, of [12, theorem 1(i)]. A stable Jacobi field along a geodesic in $H$ is any Jacobi field $Y(t)$ that can be constructed in the following way. Choose a $C^{1}$-curve $\sigma(s)$ in $L\left(\gamma^{\prime}(0)\right)$ with

$$
\sigma(0)=\gamma(0)
$$

Let $\hat{\sigma}(s)$ be the unit normal to $L\left(\gamma^{\prime}(0)\right)$ at $\sigma(s)$ pointing to the same side as $\gamma^{\prime}(0)$. Define

$$
\alpha(s, t)=\gamma_{\hat{\sigma}(s)}(t)
$$

We see from proposition 1.1(i) that $\alpha$ is $C^{1}$. Define

$$
Y(t)=\frac{\partial \alpha}{\partial s}(0, t)
$$

Note that $Y(t)$ is uniquely determined by $\sigma^{\prime}(0)$, and $Y(t) \perp \gamma^{\prime}(t)$ for all $t$, since $\sigma^{\prime}(0) \perp \gamma^{\prime}(0)$ and $\alpha$ is a variation through unit speed geodesics. Unstable Jacobi fields are obtained from $\bar{L}\left(\gamma^{\prime}(0)\right)$ in a similar way. The stable and unstable Jacobi 
fields along a geodesic in $M$ can be defined by projection from $H$. If $v \in S M$ or $S H$ write $v^{\perp}$ for the orthogonal complement of $v$ (in $T M$ or $T H$ ). Define

$$
J_{v}(t), J_{v}^{\prime}(t), \vec{J}_{v}(t), \bar{J}_{v}^{\prime}(t): v^{\perp} \rightarrow \gamma_{v}^{\prime}(t)^{\perp}
$$

so that $J_{v}(t) w$ and $\bar{J}_{v}(t) w$ are the stable and unstable Jacobi fields respectively with initial value $w \in v^{\perp}$, and $J_{v}^{\prime}(t) w, \bar{J}_{v}^{\prime}(t) w$ are their covariant derivatives along $\gamma_{v}$. Note that $J_{v}$ is not the stable Jacobi tensor $D_{v}$ of [11], [12], [13]; indeed

$$
D_{v}(t)=J_{v}(t) \circ P_{t}^{-1}
$$

where $P_{t}: v^{\perp} \rightarrow \gamma_{v}^{\prime}(t)^{\perp}$ is parallel translation. If $J: U \rightarrow V$ is a linear map between normed spaces, write

and

$$
\begin{aligned}
& \|J\|=\sup \left\{\|J u\|_{V}:\|u\|_{U}=1\right\} \\
& ((J))=\inf \left\{\|J u\|_{V}:\|u\|_{U}=1\right\}
\end{aligned}
$$

Proposition 1.2.

(i) $J_{v}(s+t)=J_{\gamma_{v}^{\prime}(s)}(t) \circ J_{v}(s)$, $\bar{J}_{v}(s+t)=\bar{J}_{\gamma_{v}^{\prime}(s)}(t) \circ \bar{J}_{v}(s)$.

(ii) $\bar{J}_{v}(-t)=J_{-v}(t)$

(iii) $J_{v}(t), J_{v}^{\prime}(t), \bar{J}_{v}(\dot{t}), \bar{J}_{v}^{\prime}(t)$ vary continuously with $v$ and $t$.

(iv) The length of a stable (unstable) Jacobi field is non-increasing (nondecreasing) with $t$.

(v) $\left\|J_{v}(t)\right\|$ is non-increasing; $\left(\left(\bar{J}_{v}(t)\right)\right)$ is non-decreasing.

(vi) Let $Y(t)$ be a Jacobi field along $\gamma_{v}$. The following are equivalent:

(a) $\|Y(t)\|$ is bounded;

(b) $Y(t)$ is parallel (i.e. $\left.Y^{\prime}(t) \equiv 0\right)$;

(c) $Y(t)$ is both stable and unstable;

(d) $J_{v}^{\prime}(0) Y(0)=\bar{J}_{v}^{\prime}(0) Y(0)$.

Proof. (i) and (ii) are clear from the above definition of $J_{v}$ and $\bar{J}_{v}$.

(iii). We show continuity of $J_{v}(t)$ and $J_{v}^{\prime}(t)$; continuity of $\bar{J}_{v}(t)$ and $\bar{J}_{v}^{\prime}(t)$ then follows from (ii). Since a Jacobi field depends smoothly on its initial value and its initial covariant derivative, it will suffice to show that $J_{v}(0)$ and $J_{v}^{\prime}(0)$ vary continuously with $v$. But

$$
J_{v}(0)=\mathrm{Id} \quad \text { for any } v,
$$

while Eschenburg and O'Sullivan [13, proposition 4] have shown that $v \rightarrow J_{v}^{\prime}(0)$ is continuous.

(iv), (v), (vi). See [8, pp. 458-459].

The other geometrical property we need to consider is the axiom of uniform visibility. This was introduced by Eberlein and O'Neill [10] as a criterion for a manifold with non-positive curvature, or more generally no focal points (or even no conjugate points [5]), to be like a manifold with negative curvature. The idea of uniform visibility is that far away things in the universal cover $H$ should appear uniformly small. Precisely:

$M$ satisfies uniform visibility if it has no conjugate points, and for each $\varepsilon>0$ there is $R(\varepsilon)>0$ so that if $p \in H$ and $\gamma$ is a geodesic in $H$ with the distance $d(p, \gamma) \geq R(\varepsilon)$, then $\gamma$ subtends an angle $\leq \varepsilon$ at $p$. 
Now consider the Lyapunov exponents of the geodesic flow. Given a Riemannian metric on $S M$ we can define, for non-zero $\xi \in T_{v} S M$, the characteristic exponent of the vector $\xi$ at $v$,

$$
\chi^{+}(v, \xi)=\limsup _{t \rightarrow \infty} 1 / t \log \|T \Phi, \xi\| .
$$

Since $S M$ is compact this definition is independent of the choice of metric on $S M$. The natural choice is the Sasaki metric which we now describe briefly; see [14], [20] for more detail.

Let $\pi: S M \rightarrow M$ be the projection and $K: T T M \rightarrow T M$ the connector map for the Riemannian connection of $M$. It can be checked that $K: T_{v} S M \rightarrow v^{\perp}$ and the map

$$
i_{v}: T_{v} S M \rightarrow T_{\pi v} M \oplus v^{\perp}, \quad i_{v}(\xi)=(T \pi \xi, K \xi),
$$

is a linear isomorphism. For $\xi, \eta \in T_{v} S M$ define

$$
\langle\xi, \eta\rangle=\langle T \pi \xi, T \pi \eta\rangle+\langle K \xi, K \eta\rangle
$$

so $i_{v}$ becomes an isometry. The Riemannian volume defined by the Sasaki metric is the Liouville measure, $\mu$. The geodesic flow is the flow of the vector field

$$
\Xi(v)=i_{v}^{-1}(v, 0) \text {. }
$$

Let $T^{\|} S M$ be the subbundle of TSM spanned by $\Xi$, and $T^{\perp} S M$ its orthogonal complement with respect to the Sasaki metric. Both $T^{\|} S M$ and $T^{\perp} S M$ are $T \Phi_{r}$ invariant.

There is a natural bijection between $T_{v} S M$ and the Jacobi fields along $\gamma_{v}$ that arise from variations through unit speed geodesics. If $\xi \in T_{v} S M$ then

$$
Y_{\xi}(t)=T \pi \circ T \Phi_{t}(\xi)
$$

is a Jacobi field along $\gamma_{v}$, and

$$
Y_{\xi}^{\prime}(t)=K \circ T \Phi_{t}(\xi)
$$

One can show that $\xi \in T_{v}^{\|} S M$ if and only if $Y_{\xi}(t)$ is a constant multiple of $\gamma_{v}^{\prime}(t)$. If $\xi \in T_{v}^{\downarrow} S M$ then

$$
Y_{\xi}(t) \text { and } Y_{\xi}^{\prime}(t) \in \gamma_{v}^{\prime}(t)^{\perp} \quad \text { for all } t ;
$$

and conversely. Write $X_{v}^{s}, X_{v}^{u}$ for the subspaces of $T_{v} S M$ corresponding to the stable and unstable Jacobi fields respectively. We see from proposition 1.2 that $X^{s}$ and $X^{u}$ are continuous, $T \Phi_{t}$-invariant subbundles of $T^{\perp} S M$. The following lemma must be well known and has been proved in a special case in $[19$, p. 801].

LEMMA 1.3. With the above notation,

$$
\chi^{+}(v, \xi)=\limsup _{t \rightarrow \infty} 1 / t \log \left\|Y_{\xi}(t)\right\| .
$$

Proof. It is clear from the definition of the Sasaki metric that

$$
\lim \sup t^{-1} \log \left\|Y_{\xi}(t)\right\| \leq \chi^{+}(v, \xi) \text {. }
$$

To prove the reverse inequality it will suffice to show that each $\xi \in T_{v} S M$ has the following property: there is $\boldsymbol{A}(\xi)>0$ such that

$$
\left\|Y_{\xi}^{\prime}(t)\right\| \leq A(\xi)\left\|Y_{\xi}(t)\right\|
$$

for all large enough $t$. Since $T^{\|} S M$ and $T^{\perp} S M$ are orthogonal, the property for 
general $\xi$ will follow if we prove it in the two cases $\xi \in T_{v}^{\|} S M$ and $\xi \in T_{v}^{\perp} S M$. If $\xi \in T_{v}^{\|} S M$ the property is clear since $Y_{\xi}^{\prime}(t) \equiv 0$. So assume $\xi \in T_{v}^{\perp} S M$. Then we can write

$$
\boldsymbol{\xi}=\eta+\zeta
$$

where $\eta \in X_{v}^{s}, \zeta \in T_{v}^{\perp} S M$ and $Y_{\eta}(0)=Y_{\xi}(0), Y_{\zeta}(0)=0$. Eberlein has proved that both $\eta$ and $\zeta$ have the property, $[8$, propositions $2.11,2.7]$. Since $Y_{\eta}(t)$ and $Y_{\zeta}(t)$ need not be orthogonal it is not immediately clear that the property follows for $\xi$. But, unless $\zeta=0,\left\|Y_{\zeta}(t)\right\| \rightarrow \infty$ as $t \rightarrow \infty$ [8, proposition 2.9], while $\left\|Y_{\eta}(t)\right\|$ is bounded as $t \rightarrow \infty$ by proposition 1.2 (iv). Using this it is easy to see that when $\zeta \neq 0$ we can take

$$
A(\xi)=A(\zeta)+\varepsilon
$$

for any $\varepsilon>0$.

Pesin's theory studies those $v \in S M$ at which $T \Phi_{t}$ has hyperbolic behaviour. Define

$$
\Lambda=\left\{v \in S M: \chi^{+}(v, \xi) \neq 0 \quad \text { for all non-zero } \xi \in T_{v}^{\perp} S M\right\} .
$$

(This is a slight change from Pesin's definition to allow for the fact that $\chi^{+}(v, \xi)=0$ whenever $\xi \in X_{v}^{s} \oplus T_{v}^{\|} S M \backslash X_{v}^{s}$.)

We see from proposition 1.2 (iv) and lemma 1.3 that

$$
\chi^{+}(v, \xi) \leq 0 \quad \text { if } \xi \in X_{v}^{s}
$$

and

$$
\chi^{+}(v, \xi) \geq 0 \quad \text { if } \xi \in X_{v}^{u}
$$

$v \in \Lambda$ if and only if both these inequalities are always strict.

The set $\Lambda$ is intimately connected with the ergodic properties of the geodesic flow. The main result of $[\mathbf{1 8}]$ is:

THEOREM (PESIN). Let $M$ be a compact Riemannian manifold without focal points, satisfying the axiom of uniform visibility. Define $\Lambda$ as above, and let $\mu$ be the Liouville measure on SM. Then either

$$
\mu(\Lambda)=0 \text { or } \mu(\Lambda)=\mu(S M) .
$$

In the latter case the geodesic flow is ergodic with respect to $\mu$ and is isomorphic to a Bernoulli flow.

The purpose of this paper is to prove:

Theorem. Let $M$ and $\Lambda$ be as in Pesin's theorem. Then

$$
\mu(\Lambda)>0 \text {. }
$$

COROLlARY. The geodesic flow on SM is Bernoulli with respect to the Liouville measure.

The first step in the proof is to show that the closed orbits of the geodesic flow are dense in $S M$ whenever $M$ satisfies uniform visibility. This is done in $\S 2$. The proof of the theorem is completed in $\S 3$. The main idea is to show that $\Lambda$ must contain at least one closed orbit. 


\section{Uniform visibility and density of closed orbits}

Eberlein [5] has shown that if $M$ satisfies the axiom of uniform visibility then the geodesic flow on $S M$ has a dense orbit. We now show that a geodesic in $H$ which corresponds to a dense orbit in $S M$ is the only geodesic between its two endpoints in $H(\infty)$.

LEMMA 2.1. Assume $M$ satisfies uniform visibility. Let $\gamma$ be a geodesic in $H$ such that

$$
\left\{\phi_{*} \gamma^{\prime}(t): t \geq 0, \phi \in \pi_{1}(M)\right\}
$$

is dense in $\mathrm{SH}$. Then the only geodesics in $H$ biasymptotic to $\gamma$ are translates of $\gamma$.

Proof. Write $B=B\left(\gamma^{\prime}(0)\right)$. We wish to show that $B=\{\gamma(0)\}$. We show firstly that $B$ is compact. If $p \in B$, the geodesic segment joining $p$ to $\gamma(0)$ lies in $B$ (proposition 1.1(v)) and so is perpendicular to $\gamma$. Thus $\gamma$ is tangent to the geodesic ball with centre $p$ and radius $d(p, \gamma(0))$, which is strictly convex. So $\gamma(0)$ is the point on $\dot{\gamma}$ closest to $p$. Now $\gamma$ subtends angle $\pi$ at every point in $B$, so we see from uniform visibility and the above that $B$ lies in a bounded neighbourhood of $\gamma(0)$. Thus $B$ is compact.

We now show that for any points $p, q$ in $B$ there is an isometry of the convex set $B$ into itself sending $p$ to $q$. Since $B$ is compact any isometry of $B$ into itself is surjective, and so has an inverse which is also an isometry [4, p. 314]. So it will suffice to find for each $p \in B$ an isometry $\tau: B \rightarrow B$ with $\tau(\gamma(0))=p$. We shall use:

Sublemma. Suppose $C \subseteq H$ is convex and $\sigma_{n}: C \rightarrow H$ is a sequence of isometries such that $\sigma_{n}\left(c_{0}\right)$ converges for some $c_{0} \in C$. Then $\left\{\sigma_{n}\right\}$ has a pointwise convergent subsequence $\left\{\sigma_{n_{k}}\right\}$ and the map

$$
c \rightarrow \lim _{k \rightarrow \infty} \sigma_{n_{k}}(c)
$$

is an isometry of $C$ into $H$.

Proof.

$$
\sigma_{n}=\exp _{\sigma_{n}\left(c_{0}\right)}{ }^{\circ} d \sigma_{n}\left(c_{0}\right) \circ \exp _{c_{0}}^{-1}
$$

where $d \sigma_{n}\left(c_{0}\right)$ is a linear isometry of the subspace of $T_{c_{0}} H$ spanned by $\exp _{c_{0}}^{-1}(C)$ into $T_{\sigma_{n}\left(c_{0}\right)} H$. It is enough to choose $n_{k}$ so that $\left\{d \sigma_{n_{k}}\left(c_{0}\right)\right\}$ converges.

It is clear from proposition 1.1(vi) that for any $t$ the map

$$
\tau_{t}: B \rightarrow B\left(\gamma^{\prime}(t)\right)
$$

defined by translation along the geodesics biasymptotic to $\gamma$ is an isometry. Let $w$ be the unique vector in $S_{p} H$ such that $\gamma_{w}$ is biasymptotic to $\gamma$. We can choose sequences $t_{n} \rightarrow \infty$ and $\left\{\phi_{n}\right\} \subseteq \pi_{1}(M)$ so that

$$
\phi_{n *} \gamma^{\prime}\left(t_{n}\right) \rightarrow w \text {. }
$$

Applying the sublemma to $\left\{\phi_{n *}{ }^{\circ} \tau_{t_{n}}\right\}$ gives an isometry $\tau: B \rightarrow H$ with

$$
\tau(\gamma(0))=p \text {. }
$$

We see from (iv) and (iii) of proposition 1.1 that

$$
\tau(B) \subseteq B(w)=B .
$$


Finally we show that the above is impossible unless $B$ is a single point. Call $p \in B$ extreme if $p$ does not lie in the interior of a geodesic segment contained in $B$. There is at least one extreme point in $B$. For, since $B$ is compact, there is $p_{0} \in B$ as far away from $\gamma(0)$ as possible. The point $p_{0}$ is extreme since the closed geodesic ball in $H$ with centre $\gamma(0)$ and radius $d\left(p_{0}, \gamma(0)\right)$ contains $B$, is strictly convex, and has $p_{0}$ on its boundary. Clearly any isometry $\tau: B \rightarrow B$ maps non-extreme points to non-extreme points. But we know that $\tau$ has an inverse $\tau^{-1}$, which is an isometry as well. So $\tau$ must also map extreme points to extreme points. It follows that every point in $B$ is extreme. This is impossible if $B$ contains two distinct points. Hence $B=\{\gamma(0)\}$.

Call a vector in $S M$ periodic if its orbit under the geodesic flow is closed, and call a vector in $S H$ periodic if it is a lift of a periodic vector in $S M$.

COROllary 2.2. If $M$ satisfies uniform visibility the periodic vectors are dense in $S M$ and $S H$.

Proof. We use an argument of Eberlein [6, theorem 3.10]. Let $\gamma$ be as in lemma 2.1. It suffices to show that, for some $t, \gamma^{\prime}(t)$ is a limit of periodic vectors in $H$.

Suppose $\phi \in \pi_{1}(M)$. Then $\phi$ has at least one axis, that is, a geodesic $\delta$ in $H$ such that

$$
\phi \circ \delta(t) \equiv \delta(t+c)
$$

for some constant $c[11, \S 4.3]$. Note that the tangents to $\delta$ are periodic vectors and $\delta(-\infty)$ and $\delta(\infty)$ are fixed points of $\phi$ (extended to act on $\bar{H}$ ). Eberlein [5, proposition 2.6] has shown that if $M$ satisfies uniform visibility then $\phi$ has at most two fixed points in $\bar{H}$. So $\phi$ has exactly two fixed points, which must be the ends of all the axes of $\phi$.

Eberlein has also shown [5, proposition 2.8] that if $M$ satisfies uniform visibility and $x, y$ are distinct points in $H(\infty)$ then there is a sequence $\left\{\phi_{n}\right\} \subseteq \pi_{1}(M)$ so that $\phi_{n}$ has fixed points $x_{n}, y_{n}$ with

$$
x_{n} \rightarrow x \text {, and } y_{n} \rightarrow y .
$$

For each $n$ let $\delta_{n}$ be an axis of $\phi_{n}$, oriented so

If $p$ is any point in $H$,

$$
\delta_{n}(-\infty)=x_{n}, \quad \text { and } \quad \delta_{n}(\infty)=y_{n} .
$$

$$
\Varangle_{p}\left(x_{n}, y_{n}\right) \rightarrow \Varangle_{p}(x, y)>0
$$

since $x \neq y$. It follows using uniform visibility that $p$ has a compact neighbourhood through which all of the geodesics $\delta_{n}$ pass. So there is a sequence $\left\{t_{n}\right\}$ such that $\left\{\delta_{n}^{\prime}\left(t_{n}\right)\right\}$ lies in a compact subset of $S H$. Let $v$ be a limit vector of this sequence of periodic vectors. Then

$$
\gamma_{v}(-\infty)=x, \quad \text { and } \quad \gamma_{v}(\infty)=y
$$

Apply this with $x=\gamma(-\infty)$ and $y=\gamma(\infty)$. We see using lemma 2.1 that $v=\gamma^{\prime}(t)$ for some $t$. 
3. Proof of the theorem

Define

$$
\begin{array}{r}
\Lambda_{0}=\{v \in S M: \\
: \limsup _{t \rightarrow \infty} t^{-1} \log \left\|J_{v}(t)\right\|<0, \\
\left.\liminf _{t \rightarrow \infty} t^{-1} \log \left(\left(\bar{J}_{v}(t)\right)\right)>0\right\} .
\end{array}
$$

It is clear from lemma 1.3 that $\Lambda_{0} \subseteq \Lambda$.

LEMMA 3.1. If $\Lambda_{0} \neq \varnothing$, then $\mu\left(\Lambda_{0}\right)>0$.

Proof. We use an argument of Pesin $\left[19\right.$, p. 803]. Let $v_{0} \in \Lambda_{0}$. We can choose $t_{0}>0$ and $\lambda_{0}$ such that $0<\lambda_{0}<1$, with

$$
\left\|J_{v_{0}}\left(t_{0}\right)\right\|<\lambda_{0}, \quad\left(\left(\bar{J}_{v_{0}}\left(t_{0}\right)\right)\right)>\lambda_{0}^{-1} .
$$

Since $J_{v}\left(t_{0}\right)$ and $\bar{J}_{v}\left(t_{0}\right)$ vary continuously as $v$ varies in $S M$ (proposition 1.2(iii)), we can find an open neighbourhood $U$ of $v_{0}$ and $\lambda$ such that $\lambda_{0} \leq \lambda<1$, with

$$
\left\|J_{v}\left(t_{0}\right)\right\|<\lambda, \quad\left(\left(\bar{J}_{v}\left(t_{0}\right)\right)\right)>\lambda^{-1}
$$

for every $v \in U$. Recall that the Liouville measure $\mu$ is invariant under the geodesic flow. By the ergodic theorem there is a set $G \subseteq S M$ with $\mu(G)>0$ such that for any $v \in G$

$$
\liminf _{t \rightarrow \infty} 1 / t \int_{0}^{t} \chi_{U}\left(\Phi_{s}(v)\right) d s>\frac{1}{2} \mu(U)>0,
$$

where $\chi_{U}$ is the indicator function of the set $U$. So, if $v \in G$, the forward orbit of $v$ under the geodesic flow spends a positive fraction of time inside $U$ where stable Jacobi fields definitely shrink and unstable Jacobi fields expand. We see using the definition of $U$ and proposition 1.2(i), (iv) that $G \subseteq \Lambda_{0}$.

There is a simple criterion to decide whether or not a periodic vector is in $\Lambda_{0}$.

Lemma 3.2. Suppose $v \in S M$ is periodic. Then either $v \in \Lambda_{0}$ or there is a non-zero Jacobi field along $\gamma_{v}$ which is orthogonal to $\gamma_{v}^{\prime}$ and parallel (i.e. its covariant derivative vanishes).

Proof. Let $T$ be the period of $\gamma_{v}$. Then

$$
J_{v}(T): v^{\perp} \rightarrow v^{\perp} .
$$

We see using proposition 1.2 (i), (iv) that

$$
\limsup _{t \rightarrow \infty} t^{-1} \log \left\|J_{v}(t)\right\|=\limsup _{n \rightarrow \infty} n^{-1} \log \left\|J_{v}(T)^{n}\right\|=\log \lambda,
$$

where $\lambda$ is the modulus of the largest eigenvalue of $J_{v}(T)$. By proposition 1.2(iv)

$$
\left\|J_{v}(T) w\right\| \leq\|w\| \quad \text { for every } w \in v^{\perp} .
$$

So $\lambda \leq 1$. If $\lambda<1$ we have that

$$
\limsup _{t \rightarrow \infty} t^{-1} \log \left\|J_{v}(t)\right\|<0 .
$$

If $\lambda=1$, there is an invariant subspace of $v^{\perp}$ on which all the eigenvalues of $J_{v}(T)$ have modulus 1 . So on this subspace $J_{v}(T)$ is volume preserving but does not expand 
the length of any vector. It follows that $J_{v}(T)$ is an isometry on this subspace. Hence there is $w \in v^{\perp}$ with

$$
\left\|J_{v}(T)^{n} w\right\|=1 \quad \text { for every } n \in \mathbb{Z} .
$$

It follows from proposition 1.2(iv) that the stable Jacobi field $J_{v}(t) w$ has constant length 1 . It is parallel by proposition $1.2(\mathrm{vi})$.

Thus we have shown that either

$$
\limsup _{t \rightarrow \infty} t^{-1} \log \left\|\bar{J}_{v}(t)\right\|<0
$$

or there is a non-zero parallel Jacobi field orthogonal to $\gamma_{v}^{\prime}$. A similar argument shows that either

$$
\liminf _{t \rightarrow \infty} t^{-1} \log \left(\left(\bar{J}_{v}(-t)\right)\right)>0
$$

or there is a non-zero parallel Jacobi field orthogonal to $\gamma_{v}^{\prime}$. The lemma follows immediately from these two statements.

Now we use the preceding lemma and the density of the periodic vectors to show that $\Lambda_{0} \neq \varnothing$ if $M$ satisfies uniform visibility.

Lemma 3.3. Assume $M$ satisfies the axiom of uniform visibility. Then the set $\Lambda_{0}$ contains a periodic vector.

Proof. Suppose not. Then there is a non-zero parallel Jacobi field along each closed geodesic in $\boldsymbol{M}$. Using corollary 2.2 we see that there is a non-zero parallel Jacobi field along each geodesic in $H$. We use this to obtain a contradiction. Let $\gamma$ be a geodesic in $H$ such that

$$
\left\{\phi_{*} \gamma^{\prime}(t): t \geq 0, \phi \in \pi_{1}(M)\right\}
$$

is dense in $H$. We shall find a geodesic biasymptotic to $\gamma$, contrary to lemma 2.1. If $v \in S H$, let

$$
P(v)=\left\{w \in v^{\perp}: J_{v}^{\prime}(t) w \equiv 0\right\}
$$

Using proposition $1.2(\mathrm{vi})$ we see that

$$
P(v)=\operatorname{ker}\left(\bar{J}_{v}^{\prime}(0)-J_{v}^{\prime}(0)\right) .
$$

Let $k$ be the smallest value of $\operatorname{dim} P(v)$ for $v \in S H$. From the above we have $k \geq 1$. Since $\bar{J}_{v}^{\prime}(0)$ and $J_{v}^{\prime}(0)$ both vary continuously with $v$ (proposition $1.2($ iii)), the set

$$
\{v \in S H: \operatorname{dim} P(v)=k\}
$$

is open. This set is clearly invariant under covering transformations. So, by reparametrizing if necessary, we can assume that $P\left(\gamma^{\prime}(0)\right)$ has dimension $k$.

Write $L=L\left(\gamma^{\prime}(0)\right)$ and for $p \in L$ let $\hat{p}$ be the unit normal to $L$ pointing to the same side of $L$ as $\gamma^{\prime}(0)$. Since $L$ is $C^{2}$-embedded in $H$ (proposition 1.1(i)), $\hat{p}$ certainly varies continuously on $L$. Thus there is an open neighbourhood $U$ of $\gamma(0)$ in $L$ such that $P(\hat{p})$ has dimension $k$ for every $p \in U$. Using this and proposition 1.2(iii) we see that $P(\hat{p})$ is a continuous $k$-dimensional distribution on $U$. Let $X$ be a continuous vector field on $U$ with $X(p) \in P(\hat{p})$ for every $p \in U$ and $X(\gamma(0)) \neq 0$. 
For some $\varepsilon>0$ there is a $C^{1}$-curve

$$
\sigma:(-\varepsilon, \varepsilon) \rightarrow U, \quad \text { with } \quad \sigma(0)=\gamma(0),
$$

which is an integral curve of $X[15$, p. 3$]$.

Define

$$
\Sigma:(-\varepsilon, \varepsilon) \times \mathbb{R} \rightarrow H, \quad \Sigma(s, t)=\gamma_{\hat{\sigma}(s)}(t) .
$$

We see using proposition 1.1(i) that $\Sigma$ is $C^{1}$. For each fixed $s, \partial \Sigma / \partial s(s, \cdot)$ is a stable Jacobi field along $\gamma_{\hat{\sigma}(s)}$, and

$$
\partial \Sigma / \partial s(s, 0)=X(\sigma(s)) \in P(\hat{\sigma}(s)) .
$$

We see that each of the vector fields $\partial \Sigma / \partial s(s, \cdot)$ is parallel along $\gamma_{\hat{\sigma}(s)}$. It follows that for any $t$

$$
\begin{aligned}
d(\gamma(t), \Sigma(s, t)) & \leq \int_{0}^{s}\left\|\frac{\partial \Sigma}{\partial s}(s, t)\right\| d s \\
& =\int_{0}^{s}\|X(\sigma(s))\| d s,
\end{aligned}
$$

which is independent of $t$. Hence the geodesics $\gamma$ and $\boldsymbol{\gamma}_{\hat{\boldsymbol{\sigma}}(s)}$ are biasymptotic for any $s \in(-\varepsilon, \varepsilon)$. Since $X(\gamma(0)) \neq 0, \sigma(s) \neq \gamma(0)$ for small $s$, and so we have a contradiction to lemma 2.1 .

The theorem follows immediately from lemmas 3.1 and 3.3.

\section{Non-positive curvature}

It is known that if the compact manifold $M$ has non-positive curvature, then $M$ satisfies uniform visibility if and only if the universal cover $H$ contains no embedded flat totally geodesic plane [5, theorem 4.1]. Ballmann [1], [2] has studied manifolds of non-positive curvature satisfying the weaker condition that there should be at least one geodesic in $H$ which does not bound a flat totally geodesic half plane. He has shown that many of the properties of uniform visibility manifolds still hold. In particular, the geodesic flow on $S M$ is topologically transitive. We now show that the results of this paper still hold under Ballmann's condition. This result has also been obtained by Ballmann and Brin.

THEOREM 4.1. Suppose $M$ has non-positive curvature and satisfies Ballmann's condition. Then $\mu(\Lambda)=\mu(S M)$ and the geodesic flow is ergodic and Bernoulli.

Proof. First we show that $\mu(\Lambda)>0$. We need to check that lemma 2.1 and corollary 2.2 still hold when uniform visibility is weakened to Ballmann's condition. We know from [2, theorem 3.5] that the geodesic flow is still topologically transitive.

Uniform visibility was used in the proof of lemma 2.1 only in showing that $B$ was compact. In the present case we can argue as follows. If $B$ is not compact, we see using the Flat Strip Theorem (proposition 1.1(vii)) that the geodesic $\gamma$ in lemma 2.1 must bound a flat half plane. But then it follows from the density property of $\gamma$ that every geodesic in $H$ bounds a flat half plane, contradicting Ballmann's condition. 
Ballmann has shown that the properties of uniform visibility manifolds used in the proof of corollary 2.2 still hold under his condition [2, theorem 3.8].

Thus $\mu(\Lambda)>0$. In particular, there is a geodesic $\gamma$ in $M$ such that there is no non-zero parallel Jacobi field along $\gamma$ orthogonal to $\gamma^{\prime}$. By [3, theorem 1], the geodesic flow on $S M$ is ergodic and Bernoulli. Since $\Lambda$ is an invariant set of positive measure, $\mu(\Lambda)=\mu(S M)$.

This result should generalize to manifolds with no focal points.

The work in this paper is intended to form part of the author's Ph.D. thesis at the University of Warwick. The author would like to thank very sincerely his supervisor, Dr. Anthony Manning, for his advice and encouragement. He also thanks Roger Butler and Ralf Spatzier for helpful conversations, and Dr. Werner Ballmann for helpful correspondence.

\section{REFERENCES}

[1] W. Ballmann. Einige neue Resultate über Mannigfaltigkeiten nicht positiver Krümmung. Bonner Mathematische Schriften 113.

[2] W. Ballmann. Axial isometries of manifolds of non-positive curvature. Math. Annalen 259 (1982), 131-144.

[3] W. Ballmann \& M. Brin. On the ergodicity of geodesic flows. Ergodic Theory and Dynamical Systems. To appear.

[4] J. Dugundji. Topology. Allyn and Bacon: Boston, 1966.

[5] P. Eberlein. Geodesic flow in certain manifolds without conjugate points. Trans. Amer. Math. Soc. 167 (1972), 151-170.

[6] P. Eberlein. Geodesic flows on negatively curved manifolds I. Ann. of Math. 95 (1972), 492-510.

[7] P. Eberlein. Geodesic flows on negatively curved manifolds II. Trans. Amer. Math. Soc. 178 (1973), 57-82.

[8] P. Eberlein. When is a geodesic flow of Anosov type? I. J. Differential Geometry. 8 (1973), 437-463.

[9] P. Eberlein. When is a geodesic flow of Anosov type? Il. J. Differential Geometry. 8 (1973), 565-577.

[10] P. Eberlein \& B. O'Neill. Visibility manifolds. Pacific J. Math. 46 (1973), 45-109.

[11] J. H. Eschenburg. Stabilitätsverhalten des geodätischen Flusses riemannscher Mannigfaltigkeiten. Bonner Mathematische Schriften 87.

[12] J. H. Eschenburg. Horospheres and the stable part of the geodesic flow. Math. Zeitschrift. 153 (1977), 237-251.

[13] J. H. Eschenburg \& J. J. O'Sullivan. Growth of Jacobi fields and divergence of geodesics. Math. Zeitschrift. 150 (1976), 221-237.

[14] D. Gromoll, W. Klingenberg \& W. Meyer. Riemannsche Geometrie im Grossen. Springer Lecture Notes in Math. no. 55. Springer: Berlin, 1968.

[15] V. V. Nemytskii \& V. V. Stepanov. Qualitative theory of differential equations. Princeton University Press: Princeton, 1960.

[16] J. J. O'Sullivan. Riemannian manifolds without focal points. J. Differential Geometry. 11 (1976), 321-333.

[17] Ja. B. Pesin. Characteristic Lyapunov exponents and smooth ergodic theory. Russian Math. Surveys. 32 (1977), 55-114. Uspekhi Mat. Nauk. 32 (1977), 55-112.

[18] Ja. B. Pesin. Geodesic flows on closed Riemannian manifolds without focal points. Math. USSR Izvestija. 11 (1977), 1195-1228. Izv. Akad. Nauk SSSR Ser. Mat. 41 (1977), 1252-1288.

[19] Ja. B. Pesin. Equations for the entropy of a geodesic flow on a compact Riemannian manifold without conjugate points. Math. Notes. 24 (1978), 796-805. Matematicheskie Zametki. 24 (1978), 553-570.

20] S. Sasaki. On the differential geometry of tangent bundles of Riemannian manifolds. Tôhoku Math. J. II Ser. 10 (1958), 338-354. 